\title{
Signal recognition basing on optical fiber vibration sensor
}

\author{
Xu Haiyan,Zhang Zhuo,Zhang Xuewu \\ College of Computer and Information Engineering, Hohai University \\ Changzhou, China \\ e-mail:xhyjstu@hotmail.com
}

\begin{abstract}
Distributed optical fiber sensor can acquire the information of physical field along time and spatial continuous distribution. It plays an important role in long-distance oil and electricity transmission and security. In this paper, the author introduced the universal steps in triggering pattern recognition, which includes signal characteristics extracting by accurate endpoint detecting, templates establishing by training, and pattern matching. By training the samples acquired in the laboratory, three templates are established. And pattern matching had been done between templates and all the samples. The results show that, 87.5 percent of the samples are matched correctly with the triggering patterns they are belonging to.
\end{abstract}

Keywords-optic fiber; vibration sensors; pattern recognition

\section{INTRODUCTION}

In recent years, distributed fiber optic sensors have widely application in long-distance, strong-EMI condition for monitoring vibration and sound signals[1-5]. Distributed fiber optic sensors are multiplexed in a single fiber as a signal carrier and distributed sensing unit, pick up the external disturbance, re-use of spectrum analysis of the signal demodulation technique, positioning the disturbance source monitoring [6-9]. In engineering applications, the identification of the type of disturbance source enables monitoring to make correctly reflect and effective measures, thus become the urgent needs of distributed optical fiber sensing system.

There are mainly two methods in existing pattern recognition techniques of fiber vibrant sensor. One is based on the invasion of the characteristics of the vibration signals by time domain analysis, such as the vibration amplitude of the signal, or zero-crossing rate. The method due to the small number of characteristic parameters for pattern recognition, and therefore couldn't accurately distinguish various external vibration signals. The second is the signal spectrum technology, as a basis for discrimination based on multidimensional spectrum characteristics. The method is computationally intensive, complex algorithms, and poor real-time. Meanwhile the distributed fiber optic sensor signal is based on optical fiber interferometer formed and can't be a good fit for the existing model.

This paper provides short-term energy and zero-crossing rate as the two kinds of the endpoint detection technology and a DTW-based approach to extract features of the sensing signals, which could be used for pattern recognition in real project, and the approach is proved by large practical experiments and projects.

\section{SYSTEM STRUCTUER AND THEORY}

A. Introduction of the System

The system is designed as follows (Fig. 1). It includes: a light source with short-coherent length(SLD), a $3 \times 3$ coupler, a $2 \times 2$ coupler, a Faraday rotator mirror(FRM), some single mode fiber (delay arm and direct arm), two PINs.

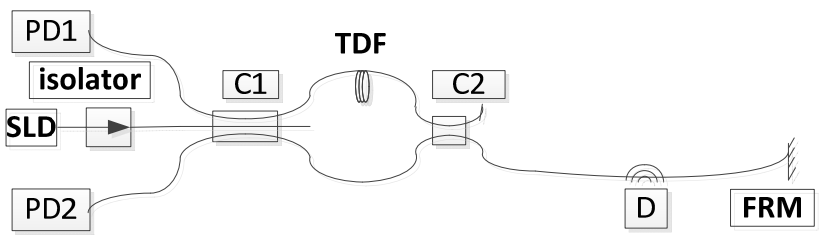

Fig. 1 Schematic diagram of the system

In Figure 1, a continuous-wave light emitted from the SLD enters into the isolator, passing through a $3 \times 3$ coupler, at the output of which the light is divided into clockwise (CW) path and counter clockwise (CCW) path as follows:

1) $1 \mathrm{a} 1-1-1 \mathrm{~b} 1-5-2 \mathrm{~b} 1-2 \mathrm{a}-7-13-7-2 \mathrm{a}-2 \mathrm{~b} 2-1 \mathrm{~b} 2-1$

2) $1 \mathrm{a} 1-1-1 \mathrm{~b} 2-2 \mathrm{~b} 2-2 \mathrm{a}-7-13-7-2 \mathrm{a}-2 \mathrm{~b} 1-5-1 \mathrm{~b} 1-1$

The two paths have the same length, the two counter propagating beams can stably interfere at the $3 \times 3$ coupler, the light intensity detected at the output (PD1 and PD2) of the optical fiber vibration sensor can be expressed as :

$$
\begin{aligned}
& \mathrm{E}_{2}=\mathrm{E}_{20} \exp \left\{\mathrm{j}\left[\omega_{\mathrm{c}} \mathrm{t}+\varphi\left(t-\tau_{3}\right)+\varphi\left(t-\tau_{4}\right)+\varphi_{2}\right]\right\} \\
& \mathrm{E}_{1}=\mathrm{E}_{10} \exp \left\{\mathrm{j}\left[\omega_{\mathrm{c}} \mathrm{t}+\varphi\left(t-\tau_{1}\right)+\varphi\left(t-\tau_{2}\right)+\varphi_{1}\right]\right\}
\end{aligned}
$$

Where

$$
\begin{aligned}
& \tau_{1}=\frac{n l_{0}}{c}, \tau_{2}=\frac{n\left(l_{0}+2 l_{1}\right)}{c} \\
& \tau_{3}=\frac{n\left(l_{d}+l_{0}\right)}{c}, \tau_{4}=\frac{n\left(l_{d}+l_{0}+2 l_{1}\right)}{c}
\end{aligned}
$$

Where $c$ is the speed of light, $n$ is the fiber refractive index, $\phi_{1}, \phi_{2}$ is the initial phase, $l_{0}$ is the distance between the $2 \times 2$ coupler and disturbance, $l_{1}$ is the distance between the position of the voltage discharge and the reflect mirror, $L_{d}$ is the length of the delay fiber. Let $T=2 n L / c$, the equation(1) and (2) can be also expressed as:

$\varphi(t)=\psi_{0}\{\sin [\omega(t+\tau)]+\sin [\omega(t+\tau+T)]\}-\psi_{0}\{\sin \omega t+\sin [\omega(t+T)]\}$ 


$$
=4 \psi_{0} \cos \frac{\omega T}{2} \sin \frac{\omega \tau}{2} \cos \omega\left(t+\frac{\tau+T}{2}\right)
$$

The system theory is that when the disturbance signal is imposed on the sensing optical fiber, the voltage discharge causes the change of the fiber length which then changes the refractive index of the optical fiber, thus causing the change of the phase of the light in the optical fiber. By means of the interference optical path, the change of light phase is switched to the change of light intensity.

\section{B. The extraction of the Signal Sample}

The system signal schematic is shown in Fig.2.

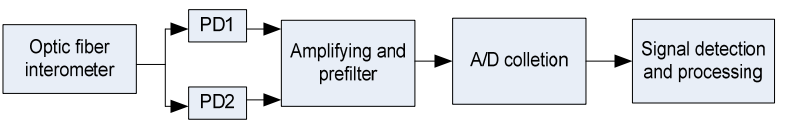

Figure 2. System signal schematic

This paper introduced short-term energy ratio as the endpoint detection technology for the interception of the useful signal in the received signals and removal silence intervals. The purpose of the endpoint detection is extracted the disturbance signal from the acquired signals. Firstly, we need to determine the starting point of the disturbance signal. We can use the method of the direct setting of the amplitude threshold for the signal which has big SNR. In order to eliminate the effect of the large value of the noise and the amplitude values of the different samples, we introduce the concept of short-term energy ratio, which can be expressed as the equation:

$$
R_{i}=\frac{E_{i+100}}{E_{i}}
$$

In equation (5), $E_{i}$ is the short-term energy of the 100 points that begins with the point i, $E_{i+100}$ is the short-term energy of the 100 points that begins with the point $\mathrm{i}+100$.

\section{Feature Extraction of the Signal}

For the specific signal we acquired from the system, each sample belongs to a model, but the duration time are varies. In this condition, if compared the corresponding points of the two samples directly, the great match distance would be attained due to the duration of the different, and the wrong conclusion of two samples of different modes would be acquired. To solve the problem, we need put the two samples in the same time scale, in this paper, the DTW algorithm is introduced.

Define the trigger signal to be recognition as $\mathrm{R}$ and the characteristic signal template as T. The matching function is defined as a collection of points:

$$
F==\{c(k), 1<k<K\}
$$

Where

$$
c(k)=(i(k), j(k))
$$

$\mathrm{K}$ is the number of the describing path point which based on template sequence $\mathrm{T}$ and recognition sequence $\mathrm{R}, c(k)$ sequence represents the mapping sequence of the template $\mathrm{T}$ to the recognition sequence $\mathrm{R}$. $j$ is the serial number of the template sequence $\mathrm{R}, \mathrm{I}$ is the serial number of the recognition sequence $\mathrm{T}$.

The Euclidean distance between the template $\mathrm{T}$ and the recognition sequence $\mathrm{R}$ can be defined as:

$$
\begin{aligned}
& d(c(k))=d(i(k), j(k) \\
& =\left\|x(i(k))-x_{t}(j(k))\right\|_{2}
\end{aligned}
$$

The smaller the value of $d$, on behalf of the corresponding point on the $\mathrm{T}$ and $\mathrm{R}$ sequence is more similar. As the cumulative distance $D_{T}$ is the smallest one, the dynamic path is optimal:

$$
D_{T}=\min \sum_{k=1}^{K} d(c(k)) \cdot w(k)
$$

Where $w(k)$ is the non-negative weight coefficient. To attain the optimal path $D_{T}$, an effective method is to use dynamic program, find point adjacent to the front from an endpoint, and then accumulate the optimal distance:

$$
D(c(k))=d(c(k))+\min (D(c(k-1)))
$$

Input two arbitrary length of the sequence, as one the template sequence $\mathrm{R}$, and the other as the identification signal sequence $\mathrm{T}$. After the operation, to complete the recognition sequence mapping to the template sequence, and to calculate the cumulative Euclidean distance of the two sequences in the mapping process as a mode discrimination basis.

\section{Reconition of the Signal}

To recognition the disturbance signal of the optical fiber vibration system, the template library which includes several trigger modes must be created. We identify the signal through computing the matching distance between the sample and the template which belongs to the template library.

The template is obtained through training, specific DTW method with a model sample stretched to a uniform time scale, stretched and then by the sample average. The template training process can be described as follows:

- Assumed that the disturbance signal is the walking signal, we acquired eight samples denote as $T_{0} \sim T_{7}$.First, put $T_{0}$ as template, then use DTW algorithm stretch $T_{1} \sim T_{7}$ to the time scale of $T_{0}$.According to the DTW algorithm, each sample will produce a minimum matching distance $d_{i}$ when matching with $T_{0}$. Then the minimum matching distance of the $T_{0}$ template can be expressed as equation : 


$$
D_{0}=\sum_{i=1}^{7} d_{i}
$$

With the same method, the minimum matching distance of other seven samples would be arrived.

- From the previous step, a one-dimensional array consist of $D_{0} \sim D_{7}$ can be attained. Selected the smallest value from this array .If $D_{0}$ is the smallest, it means the sample $T_{0}$ is the template, the rest of the sample by $T_{0}$ as a reference, and stretching to the same time scales as $T_{0}$, the optimal mode matching will be acquired. For general, assuming $D_{i}$ is the minimum value.

- For the i-th sample as the template, using the DTW algorithm to stretched the rest of the sample to the same time scale as the i-th sample.The sample after stretched is $r_{0}, r_{1}, \cdots, r_{i-1}, r_{i}=T_{i}, r_{i+1}$, $\cdots r_{7}$.

- Finally, by average the stretched samples of $r_{0} \sim r_{7}$ the template of the walking mode can be attained.

$$
R=\frac{\sum_{i=0}^{7} r_{i}}{8}
$$

\section{EXPERIMENT}

In the experiment, a $1310 \mathrm{~nm}$ super luminescent diode (SLD) is used as the light source. The optic delay line is 1 kilometer long. The optical fiber used is standard telecommunication fiber, single mode at $1310 \mathrm{~nm}$. The signals are acquired by NI DAQ card PCI-6220. The sampling rate is $10 \mathrm{KHz} / \mathrm{s}$.

Three kinds of disturbance is applied on the sensor fiber: walking, hammering and touching. The acquired signal is shown in Fig.3.

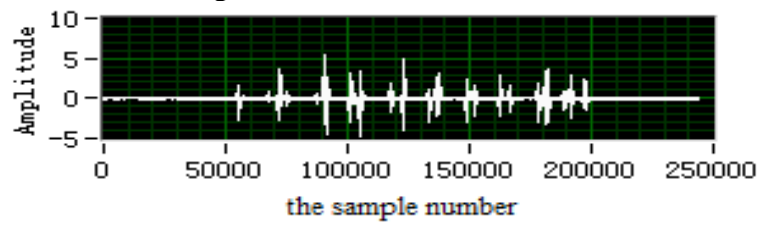

(a) Walking signals during 25 seconds

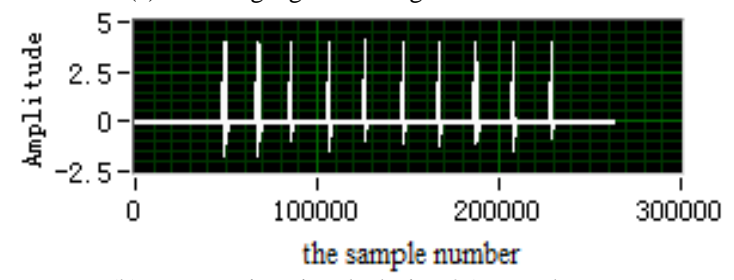

(b) Hammering signals during 25 seconds

From the above table we can see that D1 is the minimum matching distance, it means the sample $T_{1}$ is the template, the

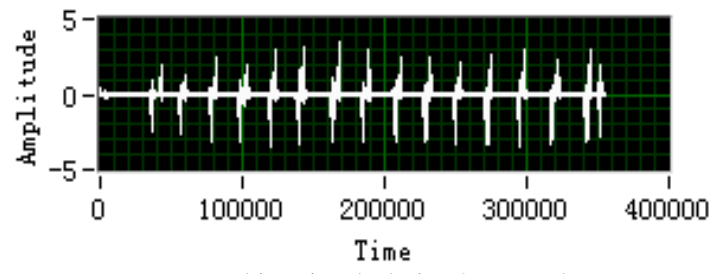

(c) Touching signals during 25 seconds

Figure 3. The signals of three modes

For the signals acquired from the sensor system, we use the method discussed above to recognition the disturbance mode. According to (5), make the threshold $R_{i}$ is 5 , (i.e. $R_{i}$ greater than 5 is the samples endpoint), as for the walking signals the extracted eight walking samples are shown in Fig.4.

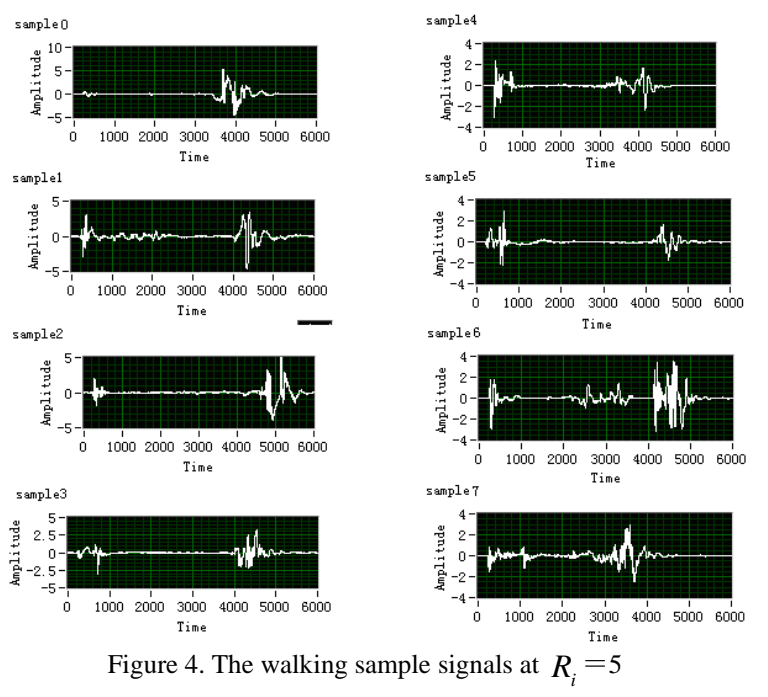

The eight walking mode signal samples during 25 seconds are extracted through the above steps. In addition a sample of two modes can be extracted by the same method.

The total minimum matching distance array after DTW for the above sample is shown in table 1 .

TABLE I. ARRAY OF THE MINIMUM MATCHING DISTURNCE

\begin{tabular}{|c|c|c|c|}
\hline D0 & D1 & D2 & D3 \\
\hline 50.044 & 34.8724 & 38.114 & 62.8739 \\
\hline D4 & D5 & D6 & D7 \\
\hline 55.2834 & 56.0522 & 48.7012 & 48.7012 \\
\hline
\end{tabular}

rest of the sample by $T_{1}$ as a reference, and stretching to the 
same time scales as $T_{1}$, the $r_{0} \sim r_{7}$ will be attained. Computing (12), template of the walking mode is shown in Fig.5(a). Defined walking mode, hammering mode and touching mode as the mode 0,1,2 respectively, the template of the rest two modes are shown in Fig.5(b),(c).

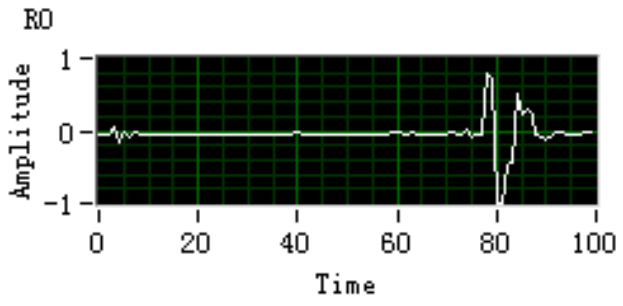

(a) Template of the walking mode after training R1

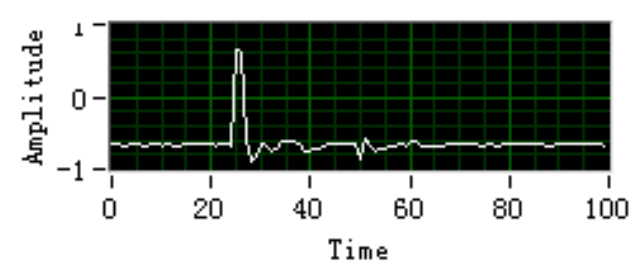

(b) Template of the hammering mode after training R3

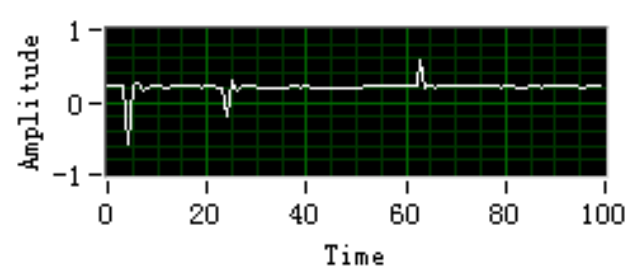

(c) Template of the touching mode after training

Figure 5. The training template of the three mode

For the other modes, using the same DWT algorithm to recognize the signal ,the pattern recognition correct rate of the various samples are shown in table 2 . The results show that, 87.5 percent of the samples are matched correctly with the triggering patterns they are belonging to.

TABLE II. RECOGNITION CORRECT RATE OF THE THREE MODES

\begin{tabular}{|c|c|c|c|}
\hline mode & walking & hammering & touching \\
\hline correct rate & $87.5 \%$ & $100 \%$ & $87.5 \%$ \\
\hline
\end{tabular}

\section{CONCLUSION}

This paper provides a DTW-based approach to extract features of the output signals of distributed vibrant sensor system, which could be used for pattern recognition in real project, and the approach is proved by large practical experiments and projects. The results show that, 87.5 percent of the samples are matched correctly with the triggering patterns they are belonging to.

\section{ACKNOWLEDGMENT}

This paper is supported by "the Fundamental Research Funds for the Central Universities (2012B03814)" and "the National Natural Sciences Foundation of China (Grant No. 61273170)”.

\section{REFERENCES}

[1] T. R. Parker, M. Farhadiroushan, V.A. Handerek, ”A Fully distributed simultaneous strain and temperature sensor using spontaneous Brillouin backscatter,” IEEE Photonics Technology Letters ,vol 9, 1997,pp.979-981.

[2] N.Fernandes, ,K.Gossner ,H. Krisch, ”Low power signal processing for demodulation of wide dynamic range of interferometric optical fibre sensor signals., ” Proc. SPIE 7653, Fourth European Workshop on Optical Fibre Sensors, 765328 (September 08, 2010).

[3] P.R.Hoffman and M.G.Kuzyk, "Position determination of an acoustic burst along a Sagnac interferometer,” J.lightw.Technol ,vol 22,2004,pp. 494-498.

[4] S.J.Russell, K.R.C.Brady, and J.P.Dakin, "Real-time location of multiple time-varying strain perturbations, acting over a $40-\mathrm{km}$ fiber section,using a novel dual-Sagnac interferometer," J.lightw.Technol,vol 19,2001,pp .205-213.

[5] H.Y.Xu,Q. Xu, Q.Xiao, B.Jia, "Disturbance Detection in Distributed Fiber-optic Sensor Using Time Delay Estimation,”Acta Optoca Sinica ,vol 30,2010,pp. 1603-1607.

[6] A.Danbridge,A.B.Tveten,T. G. Giallorenzi, "Homodyne demodulation scheme for fiber optic sensors using phase generated carrier,” IEEE Trans. Microwave Theory Tech ,vol 30 ,1982, pp.1635-1641.

[7] K.P.Koo, A.B.Tveten, and A.Dandridge, "Passive stabilization scheme for fiber interferometers using $3 \times 3$ fiber directional couplers,”Appl.Phy.letter,vol 41 ,1982,pp. 616-618.

[8] Y.Jiang,Y.M.Lou,H.W.Wang, "Software demodulation for $3 \times 3$ coupler based fiber optic interferometer," Acta Photonica Sinica, vol 27 .1998, pp.152-155.

[9] Y. Xu,Y. Q.Li, Y. Jiang, “Application of $3 \times 3$ coupler based MachZehnder interferometer in delamination patch detection in composite,” NDT \& E International,vol 44,2011,pp.469-4 